\title{
La demencia en España: evolución desigual entre las necesidades de los pacientes y la oferta asistencial desde la geriatría
}

\author{
Pedro Gila, José Regidorb, Enrique Arriolac, José Augusto García ${ }^{\mathrm{d}}$ e Isidoro Ruipérez \\ aServicio de Geriatría. Hospital Clínico San Carlos. Madrid. España. \\ 'Cátedra de Biología Celular. Facultad de Ciencias de la Salud. Universidad de Las Palmas de Gran Canaria. \\ Las Palmas. España. \\ cUnidad de Memoria y Alzheimer. Matia Fundazioa. San Sebastián. Guipúzcoa. España. \\ 'Atención Sociosanitaria y Social. Grupo Sagessa. Reus. Tarragona. España. \\ eSociedad Española de Geriatría y Gerontología (SEGG). Madrid. España.
}

Este trabajo ha sido financiado por Novartis Farmacéutica, S.A.

Correspondencia: Dr. I. Ruipérez Cantera.

Servicio de Geriatría. Hospital Central de la Cruz Roja.

Avda. Reina Victoria, 26. 28003 Madrid. España.

Correo electrónico: iruiperez.hccruzr@salud.madrid.org

\section{Resumen}

Objetivo: Estudio de la metodología empleada en el diagnóstico de las demencias en España y valoración del tratamiento más frecuente desde el punto de vista de la geriatría.

Material y método: Se realizó un censo exhaustivo, cerrado el 3110-2004, de recursos asistenciales para la atención geriátrica especializada de la demencia. Posteriormente, se realizó un estudio transversal. Los centros fueron distribuidos y validados por representantes autonómicos de la Sociedad Española de Geriatría y Gerontología (SEGG).

Resultados: En general, el programa de atención a la demencia más utilizado en España es la psicoestimulación (el 59\% de los centros), mientras que el principal protocolo específico es el de nutrición (el $81 \%$ de los centros), seguido por el de movilidad y cribado de depresión (el 75 y el $73 \%$, respectivamente). Los tratamientos farmacológicos más difundidos entre los centros españoles son los fármacos anticolinesterásicos y los neurolépticos, usados en el 33 y el $32 \%$ de los pacientes, respectivamente. Además, los principios activos específicos para la demencia más utilizados son: donepezilo, rivastigmina y memantina (el 12 , el 11 y el $8 \%$ de los pacientes, respectivamente).

Conclusiones: La atención especializada de la demencia en España presenta una alta heterogeneidad entre centros, lo que implica una variabilidad importante de la calidad asistencial que reciben los pacientes con demencias. Así, el tratamiento de los pacientes es uno de los puntos que se debe mejorar. Los hospitales son los centros en los que se trata de manera específica a los pacientes demenciados empleando anticolinesterásicos. Por el contrario, en las residencias y otros centros se tiende a utilizar fármacos neurolépticos.

Palabras clave: Demencia. Prevalencia. Geriatría. Tratamiento farmacológico.

\section{Introducción}

La demencia es un tipo de trastorno degenerativo crónico, complejo y generalmente relacionado con el envejecimiento que evoluciona de forma progresiva e irreversible. La demencia más frecuente en la sociedad occidental es la

\begin{abstract}
Objective: To study the methodology employed for the diagnosis of dementia in Spain and to evaluate the most commonly used pharmacologic treatments in geriatric services.

Material and method: We performed a descriptive, cross-sectional study. The health facilities for the specialized geriatric care of dementia in Spain were included in an exhaustive census closed on 31 st October, 2004. The centers were classified and corroborated by representatives from the autonomous communities forming part of the Spanish Society of Geriatrics and Gerontology.

Results: In general, the most frequently used therapeutic modality for dementia in Spain is psychostimulation (59\% of the centers), while the main specific protocol is nutrition ( $81 \%$ of the centers), followed by mobility and early detection of depression $(75 \%$ and $73 \%$, respectively). The main pharmacological treatment in Spanish centers is the use of acetylcholinesterase inhibitors (33\% of patients) and neuroleptic drugs (32\% of patients). The drugs most commonly used are donepezil, rivastigmine and memantine (12\%, $11 \%$ and $8 \%$ of patients, respectively).

Conclusions: The specialized care of dementia in Spain shows wide heterogeneity among centers, suggesting substantial variation in the quality of care received by these patients. Thus, the treatment of patients with dementia is one of the points that should be improved. Hospitals tend to treat patients with dementia with specific treatment consisting of acetylcholinesterase inhibitors. In contrast, nursing homes and other centers tend to use neuroleptic drugs.
\end{abstract}

Key words: Dementia. Prevalence. Geriatrics. Drug therapy.

enfermedad de Alzheimer ${ }^{1}$. Los pacientes que padecen algún tipo de demencia se incrementan cada año debido al aumento de la esperanza de vida, que en el oeste de Europa crecerá hasta los 86 años en las mujeres y 81 en los varones ${ }^{2}$. En España (quinto país del mundo con mayor proporción de personas mayores de 65 años), las perspectivas demográficas indi- 
can que el número de personas con 65 o más años aumentará del $17 \%$ (año 2000) al $20 \%$ en el año 2020, y las personas con 80 o más años llegarán a ser el $5,7 \%{ }^{2}$. Se configura así una tendencia irreversible que, según la Organización de Naciones Unidas, continuará de manera que tendrá más de 60 años 1 de cada 5 personas en el mundo en el año 2050 y 1 de cada 3 en 2150. Esta evolución demográfica implica un mayor coste tanto económico como social, ya que se incrementan el gasto sanitario y las necesidades de atención especializada.

La demencia es un síndrome adquirido que da lugar a una progresiva pérdida de memoria, así como de otras funciones cognitivas, en grado suficiente para afectar considerablemente a la calidad de vida del paciente ${ }^{3}$. La importancia del deterioro cognitivo va en aumento debido a los progresivos envejecimiento y dependencia de la población (en España se prevé que en el año 2010 habrá más de 1,5 millones de personas dependientes mayores de 65 años), lo que se refleja en los resultados de estudios epidemiológicos de nuestro entorno, que presentan una prevalencia de deterioro cognitivo entre el 8,7 y el $30,5 \%$ de los sujetos no institucionalizados y llega hasta el $56 \%$ de los sujetos institucionalizados ${ }^{4}$. Las personas con deterioro cognitivo no sólo consumen más servicios, sino que tienen la percepción de una mayor necesidad de recibirlos y piensan que actualmente no les son prestados ${ }^{5}$.

En la actualidad, España no tiene garantizada una adecuada asistencia geriátrica especializada en la mayoría de sus hospitales generales, ya que únicamente el $20 \%$ de ellos cuenta con algún geriatra. Además, la distribución de los recursos geriátricos es muy desigual entre sus diferentes comunidades autónomas ${ }^{6}$.

Las demencias, aún siendo afecciones frecuentes en personas de edad avanzada, son muy difíciles de diagnosticar de forma precisa. En general, el diagnóstico se basa en síntomas clínicos en la esfera cognitiva, como pueden ser alteraciones del comportamiento o pérdida de la memoria inmediata7. El diagnóstico final puede confirmarse mediante un estudio histológico post mórtem del cerebro del paciente ${ }^{8,9}$. Debido a la complejidad de los trastornos relacionados con la demencia, se hace muy complicado establecer un tratamiento farmacológico. Actualmente, los fármacos más utilizados para el tratamiento de la enfermedad de Alzheimer son los inhibidores de la acetilcolinesterasa (IACE) (donepezilo, rivastigmina, galantamicina o galantamina), aunque existen otros posibles tratamientos farmacológicos como la memantina, así como moléculas con propiedades antioxidantes (selegilina 0 vitamina $\mathrm{E})^{10}$.

Debido a la creciente necesidad de atención especializada de la sociedad actual, el Grupo de Demencias de la Sociedad Española de Geriatría y Gerontología (SEGG), con la colaboración de Novartis Farmacéutica S.A., impulsó el proyecto DECADA-G (Demencias en España: Calidad Asistencial y Demanda Atendida por la Geriatría). El principal objetivo de este proyecto es el análisis de la actual atención especializada en geriatría de los pacientes con demencia en España. En este trabajo se realizó un estudio detallado del proceso de diagnóstico y tratamiento de los pacientes demenciados españoles, con el fin de comparar los resultados obtenidos y evaluar posibles elementos de mejora.

\section{Material y método}

En el estudio DECADA-G se elaboró un censo de dispositivos asistenciales con atención especializada en geriatría, que incluía desde residencias geriátricas a hospitales y otros centros (centros sociosanitarios, centros de día psicogeriátricos, clínicas psicogeriátricas, centros tutelados y programas/servicios de atención domiciliaria). Este censo se realizó con el propósito de analizar las diferencias geográficas en el grado y el tipo de la cobertura geriátrica de la demencia en España durante el último año. Posteriormente se realizó un estudio transversal para la caracterización detallada de la oferta asistencial y el perfil de los pacientes con demencia atendidos. El estudio se llevó a cabo en dos fases:

Primera fase. Se elaboró una base de datos preliminar, de tipo general, con todos los hospitales, residencias geriátricas, centros sociosanitarios y otros tipos de centros. Los datos se obtuvieron de las páginas web del Ministerio de Sanidad y Consumo, el IMSERSO y el CSIC. A partir de estos datos generales se realizó un catálogo de centros que podrían participar en el estudio. Los datos del catálogo de centros fueron clasificados por comunidades autónomas y enviados a los representantes autonómicos (presidentes o vocales) de la SEGG para analizarlos, corroborarlos y eventualmente ampliarlos. Los criterios para validar e incluir los centros hospitalarios fueron: contar con al menos un médico especialista en geriatría y/o disponer de algún servicio que reuniera las siguientes condiciones: a) consultas externas realizadas por un especialista en geriatría; b) unidad de valoración supervisada por un especialista en geriatría; $c$ ) hospital de día supervisado por un especialista en geriatría; d) camas de pacientes agudos supervisadas por un especialista en geriatría, y e) unidad de media estancia (rehabilitación, convalecencia, etc.) supervisada por un especialista en geriatría. En el caso de las residencias geriátricas y los otros centros, el único criterio que se impuso fue que contaran con algún médico especialista en geriatría.

Segunda fase. En los centros validados se realizó un estudio transversal descriptivo de la oferta asistencial de los centros, así como de los pacientes con demencia atendidos. Se propuso la participación en el estudio a todos los centros validados ( $n=242$ ), aunque finalmente se obtuvo datos de un total de 119 centros, que proporcionaron información de un total de 2.319 pacientes con demencia.

Merece comentario que los 123 centros que declinaron participar en la segunda fase del proyecto presentan una distribución territorial ( $p=0,32)$ y un tipo $(p=0,13)$ homogéneos respecto a los incluidos en el estudio.

\section{Resultados}

El censo inicial, correspondiente a la primera fase, contó con un total 5.621 centros, de los que 242 cumplían los criterios de inclusión, es decir, que disponían de un médico especialista en geriatría. En España, por tanto, sólo el 4,3\% 
Figura 1. Mapa de la distribución de los centros asistenciales incluidos en el estudio DECADA-G y distribuidos por comunidades autónomas.

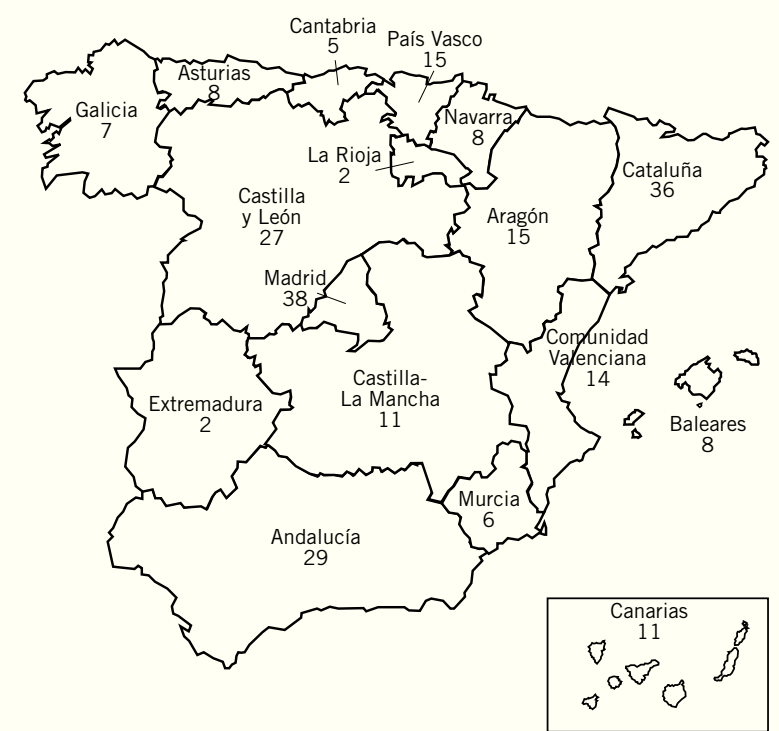

de los dispositivos asistenciales considerados cuenta con atención especializada en geriatría para la demencia. Además, según el tipo de dispositivo asistencial que se considere, el porcentaje varía significativamente. Así, contaban con algún especialista en geriatría un $20 \%$ de los hospitales, un $3 \%$ de las residencias geriátricas y un $2 \%$ de los demás centros $^{6}$. De todos los centros que podían participar en el estudio DECADA-G, 169 (70\%) aceptaron formar parte y, finalmente, 119 (49\%) enviaron datos (fig. 1).

La figura 2 muestra los porcentajes de utilización de los diferentes programas específicos de atención a la demencia ofertados en los centros españoles. En general, el programa más ampliamente utilizado es la psicoestimulación. Este programa se usa en el $59 \%$ de los centros, seguido por la terapia de orientación a la realidad, presente en un $55 \%$ de los centros (fig. 2A). Cabe destacar que los hospitales son los centros donde se utiliza menor proporción de este tipo de programas, que se oferta en más del $45 \%$ de éstos (fig. 2B). La psicoestimulación fue el programa con mayor uso hospitalario (el $44 \%$ de los hospitales), el segundo programa más utilizado fue la intervención para el control de los síntomas no cognitivos (el 38\% de los hospitales). Por otra parte, la categoría "otros centros" fue la que presentó una mayor oferta de programas para la demencia. Los programas de psicoestimulación y terapia de orientación a la realidad estaban presentes en un $93 \%$ de los "otros centros". Un dato interesante es que un $19 \%$ de los centros españoles declaró no disponer de ninguno de los programas contemplados en este estudio. Este dato mostró importantes diferencias en función del tipo del centro. Por una parte, el $33 \%$ de los hospitales declaró no disponer de ninguno de los programas del estudio, mientras que sólo el 5\% de las residencias lo declaró.

En la figura 3 se presentan los porcentajes de utilización de los diferentes protocolos específicos de atención a la demencia. En general, el estudio DECADA-G demostró que el protocolo utilizado más frecuentemente en los centros españoles es el de nutrición, presente en un $81 \%$ de los centros estudiados (fig. 3A). Después del protocolo de nutrición, los más utilizados son el protocolo de movilidad (75\%) y el de cribado de depresión (73\%). Aunque la utilización de estos protocolos es bastante uniforme entre los diferentes tipos de centros estudiados, se pudo constatar algunas diferencias (fig. 3B). En los hospitales, el protocolo con mayor porcentaje de utilización fue el de cribado de depresión (el $72 \%$ de los centros). El protocolo de nutrición mostró también una elevada utilización en este tipo de centros (el $70 \%$ de los centros), similar al de cribado de la depresión. En las residencias, de forma concordante con los datos generales, el protocolo de nutrición fue el más utilizado (93\%). Los protocolos de movilidad y de psicomotricidad también son los usados en la mayoría de las residencias estudiadas (el 85 y el $80 \%$, respectivamente). Finalmente, el $100 \%$ de los centros catalogados como "otros centros" utilizaban el protocolo de movilidad. Además, el 93\% de estos centros utilizan también los protocolos de nutrición y de estimulación de las actividades de vida diaria.

Un dato interesante es la proporción de centros asistenciales españoles que cuentan con algún tipo de programa de apoyo a los familiares de pacientes demenciados. En general, son tan sólo el 34\%. Cabe destacar que sólo el $25 \%$ de los hospitales ofrecen a los familiares de pacientes con demencia algún programa de apoyo.

A partir de los datos obtenidos en el estudio transversal, se observó que un $42 \%$ de los pacientes que presentan algún tipo de demencia están tratados farmacológicamente. De éstos, el 30\% toma un único fármaco, mientras que el $56 \%$ toma dos o más fármacos relacionados con la demencia (tratamientos específicos, neurolépticos, hipnóticos, ansiolíticos, etc.). Un $14 \%$ de los pacientes no reciben ningún fármaco relacionado con el tratamiento para la demencia. La duración de los tratamientos suele ser superior a 1 año, excepto en los casos del haloperidol y la quetiapina, que mayoritariamente son inferiores a 6 meses. La mayor parte de los pacientes con demencia incluidos en el estudio fueron tratados con inhibidores colinesterásicos (33\%) y con neurolépticos (32\%) (fig. 4). El tratamiento farmacológico de elección varía según el tipo de centro. En los hospitales (fig. 4B), los fármacos más utilizados son los inhibidores colinesterásicos, que se usan en el $38 \%$ de los pacientes. Por otra parte, en las residencias y otros centros, los fármacos más usados son los neurolépticos (el 31 y el $40 \%$ de los pacientes, respectivamente).

En la figura 5 se muestran los principales tratamientos farmacológicos que reciben los pacientes demenciados en España, según la escala de deterioro global (Global Deterioration Scale $\left.[G D S]^{11}\right)$. Si se relaciona el estadio GDS con tratamiento o ausencia de tratamiento para la demencia, se observa que en los estadios 6 o 7, un $66 \%$ de los pacientes no recibe ningún tipo de tratamiento farmacológico específico. Del total de pacientes con GDS 6-7 que están tratados 
Figura 2. Programas específicos de atención a la demencia utilizados en los centros asistenciales españoles.

A

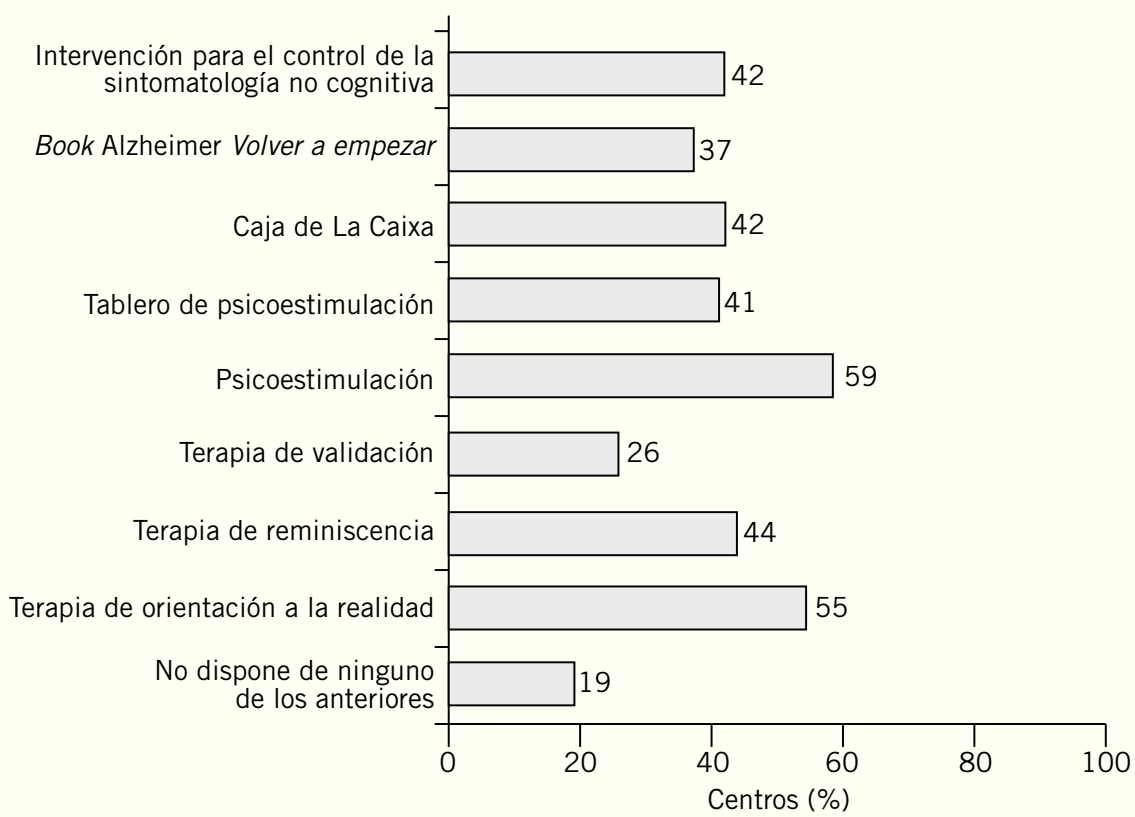

B

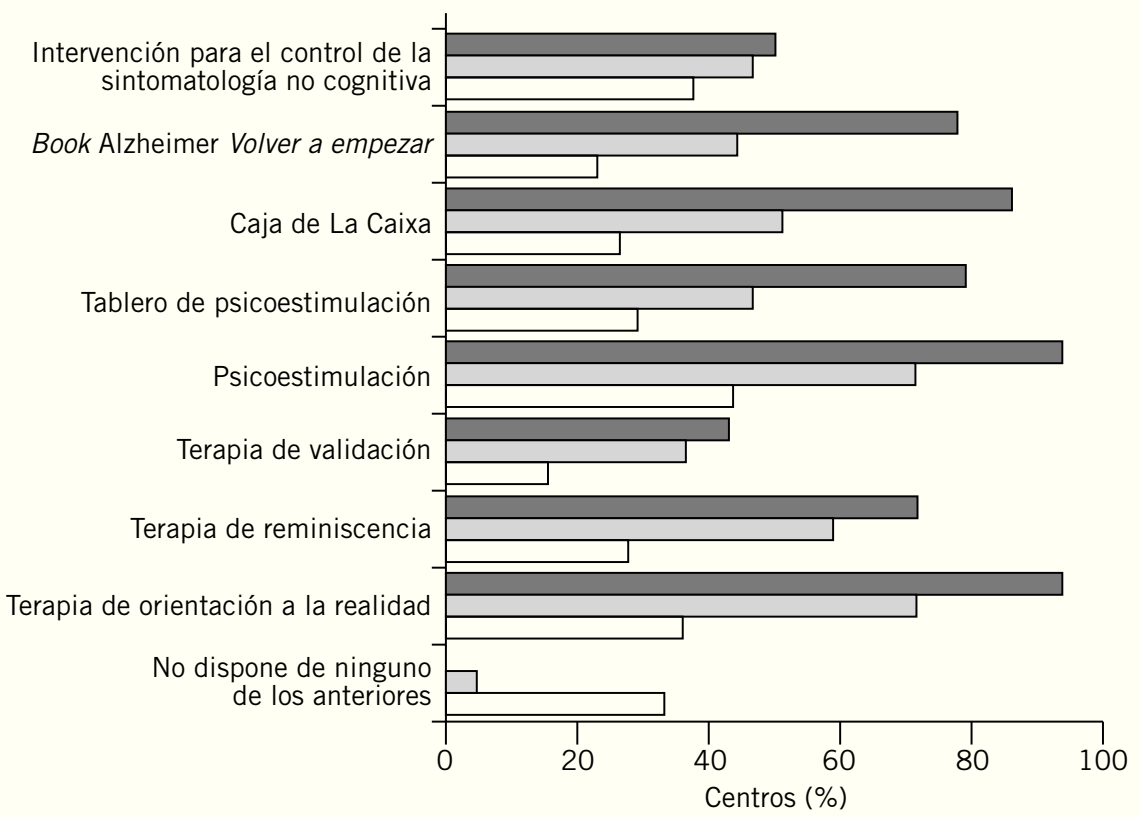

Hospitales $\square$ Residencias $\square$ Otros centros

A: distribución general; B: distribución según tipo de centro.

farmacológicamente, un $36 \%$ recibe memantina. Por otra parte, en los estadios 3,4 y 5 , el $46 \%$ de los pacientes reciben tratamiento para la demencia, y los IACE son los más empleados (el $24 \%$ de los pacientes).

Finalmente, en la figura 6 se presentan los porcentajes de utilización de algunos fármacos específicos para la de- mencia. Entre ellos, el donepezilo es el más comúnmente utilizado (un $12 \%$ de los pacientes), seguido por la rivastigmina (un $11 \%$ de los pacientes demenciados) y la memantina (8\%). Raramente se utiliza la combinación formada por inhibidores colinesterásicos y memantina, ya que sólo un $0,1 \%$ de los pacientes reciben esta combinación. 
Gil P et al. La demencia en España: evolución desigual entre las necesidades de los pacientes y la oferta asistencial desde la geriatría

Figura 3. Protocolos específicos de atención a la demencia utilizados en los centros asistenciales españoles. A: distribución general. B: distribución según tipo de centro.

A

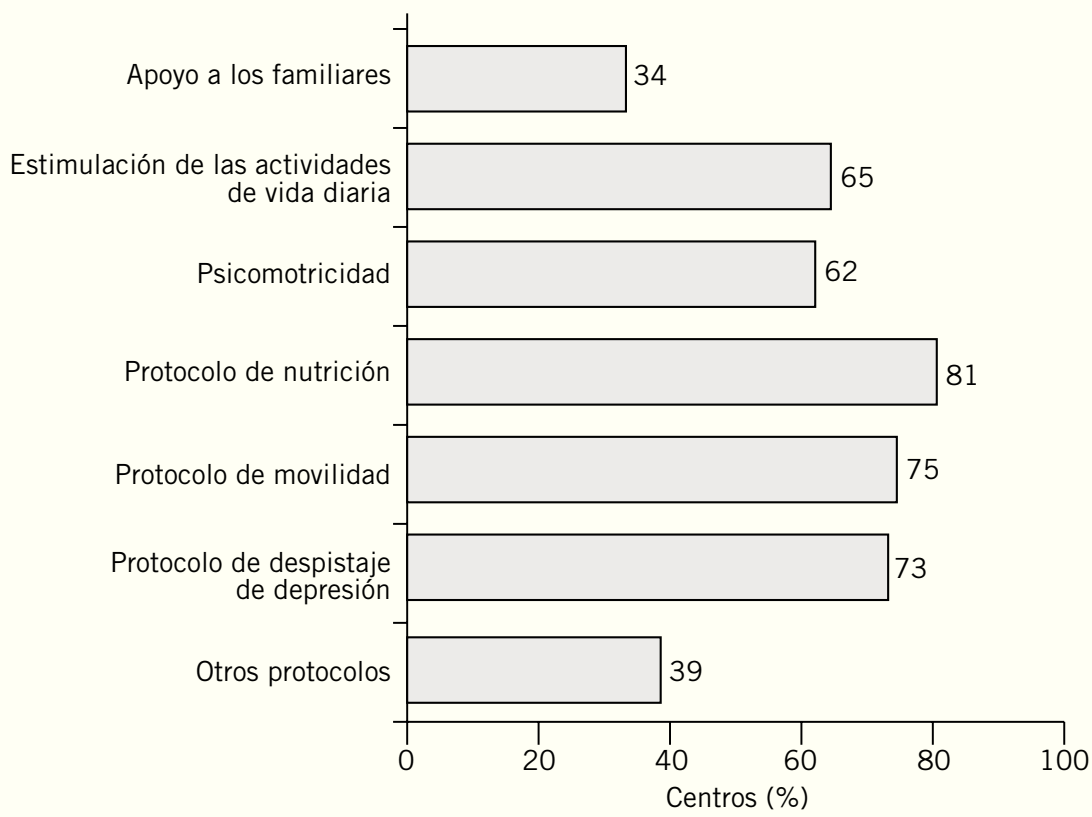

B

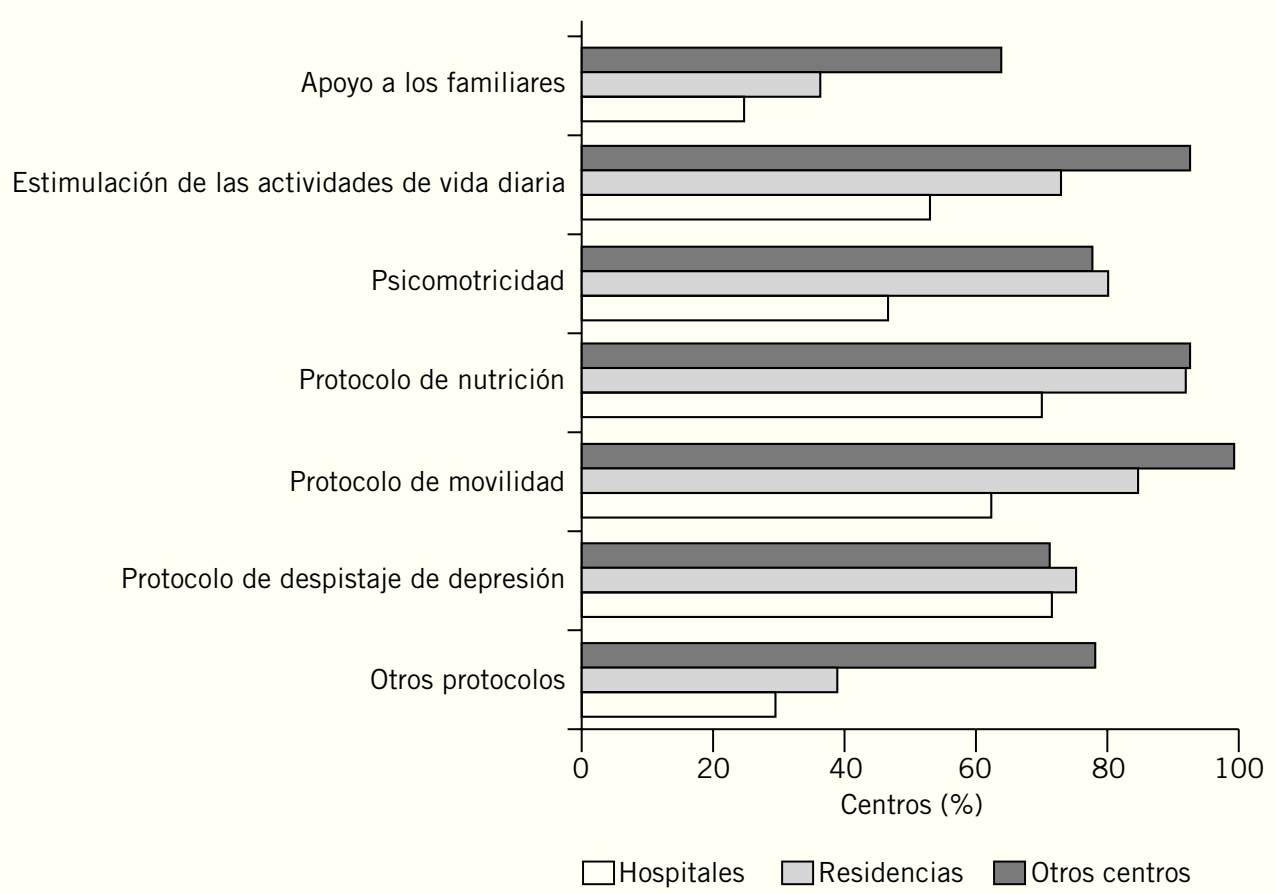

\section{Discusión}

Se llevó a cabo un estudio transversal en una muestra de centros españoles en los que se realiza una atención especializada en geriatría a pacientes con demencia. El

50,8\% declinó participar en esta fase de la investigación; sin embargo, su distribución territorial y su tipología no difieren de forma significativa de los centros analizados, por lo que no es posible objetivar ningún sesgo de selección. 


\section{Figura 4. Tratamientos farmacológicos utilizados en los centros españoles para tratar a pacientes demenciados.}

A

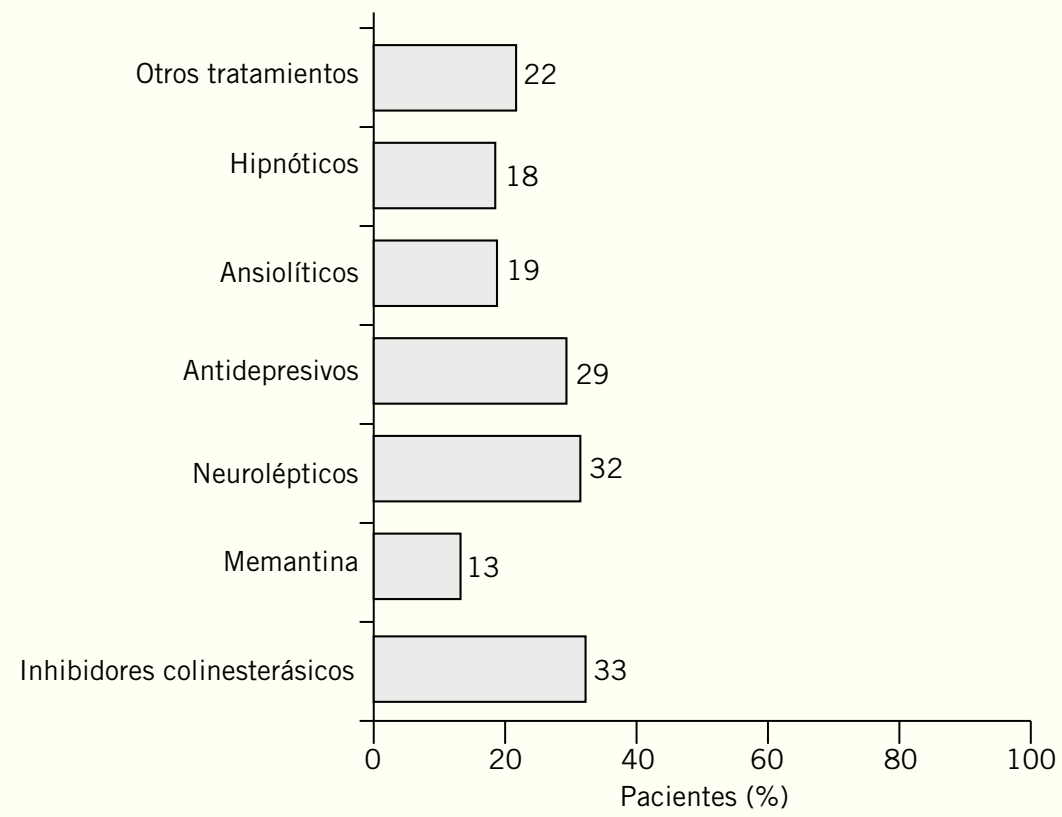

B

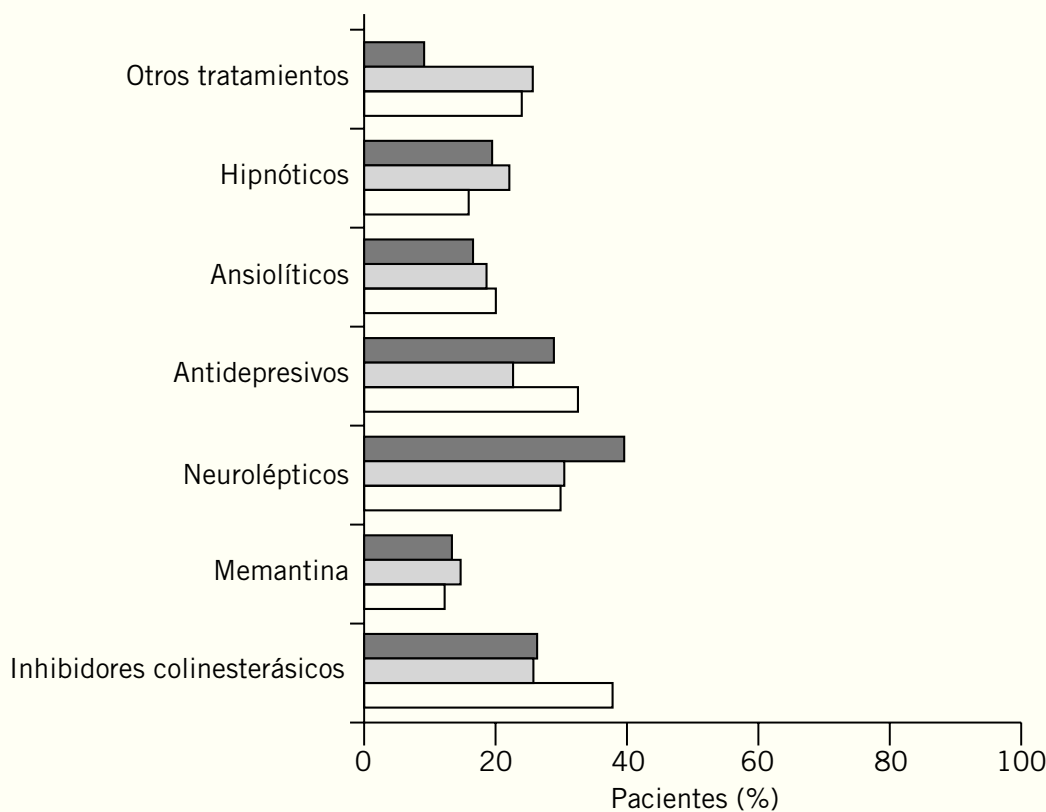

$\square$ Hospitales $\square$ Residencias $\square$ Otros centros

A: distribución general; B: distribución según tipo de centro.

En la mayoría de los centros participantes en la investigación se utiliza el programa de psicoestimulación como principal programa específico de atención a la demencia, independientemente del tipo de centro. Es destacable que son los centros hospitalarios los que presentan una menor oferta de programas específicos (menos de la mitad de los centros), aunque son precisamente éstos los que presentan una mayor especialización ${ }^{6}$. Éstos son, además, los centros que presentan el menor porcentaje de protocolos de apoyo a los familiares de pacientes demenciados, mientras que los centros diferentes de hospitales o residencias son los mejor dotados en cuanto a este tipo de protocolos, versados en la asistencia so- 
Figura 5. Tratamientos farmacológicos utilizados para tratar a pacientes demenciados*.

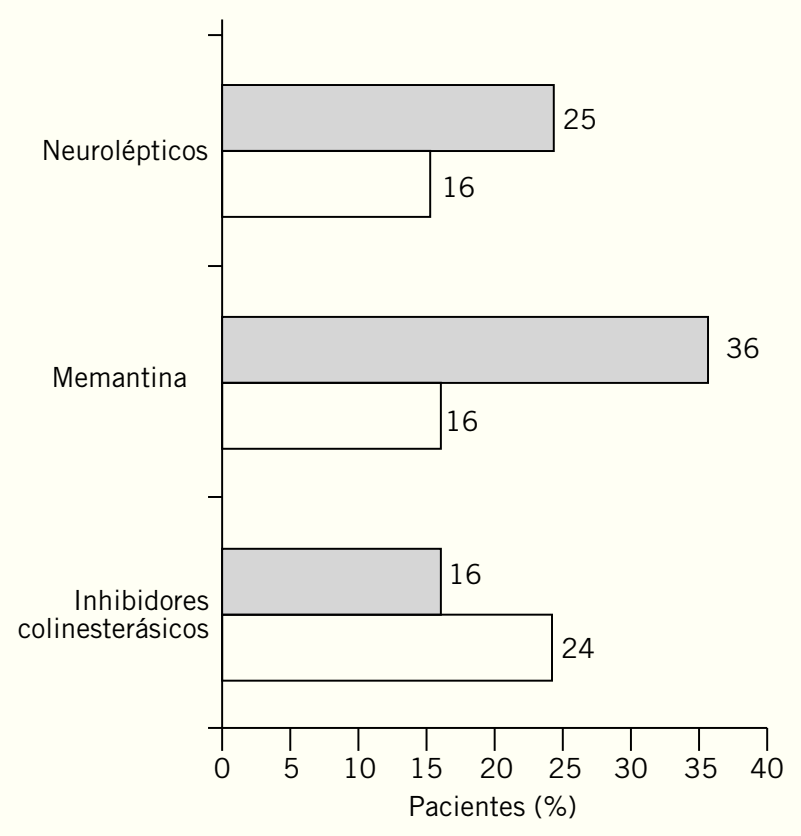

GDS 3,4 05

GDS $6 \circ 7$

*Según su estadio en la Global Deterioration Scale (GDS).

cial. En general, sólo un $34 \%$ de los centros disponen de algún programa formal de apoyo a los familiares, lo que indica un muy bajo grado de implementación de estos programas, que son de gran ayuda para los familiares de los pacientes con demencia. Existen algunas iniciativas, como es el caso de la Comunidad de Castilla y León, en las que se reconoce a la familia del demenciado como el "eje vertebral y núcleo fundamental de protección y atención a estas personas"12, Io que indica la necesidad de formar e informar a los familiares de las personas con demencia para su adecuado cuidado. Esto sería indicativo de la manifiesta necesidad de un incremento de la demanda de programas de atención a las familias en los centros en los que se atiende a personas con algún tipo de demencia.

En lo que respecta al tratamiento de los pacientes que presentan algún tipo de demencia, se recomienda utilizar el tratamiento farmacológico junto con el no farmacológico, es decir, en terapia combinada ${ }^{13}$. Según el estudio DECADA-G, menos de la mitad de los pacientes demenciados reciben algún tipo de tratamiento farmacológico, y más de la mitad de éstos se encuentran polimedicados. El tratamiento de elección más difundido entre los centros participantes es con inhibidores colinesterásicos y neurolépticos aunque, lógicamente, las características de los planes terapéuticos varían en función de la GDS.

Los tratamientos farmacológicos de las demencias deben dirigirse hacia las dianas concretas que causan los síntomas. De esta forma, las demencias que cursan con déficit de acetilcolina deben ser tratadas con IACE, independientemente del tipo de demencia ${ }^{14}$. En lo que respecta a los principios activos, el donepezilo y la rivastigmina son los más utilizados.

Figura 6. Principales principios activos utilizados en el tratamiento específico de la demencia.

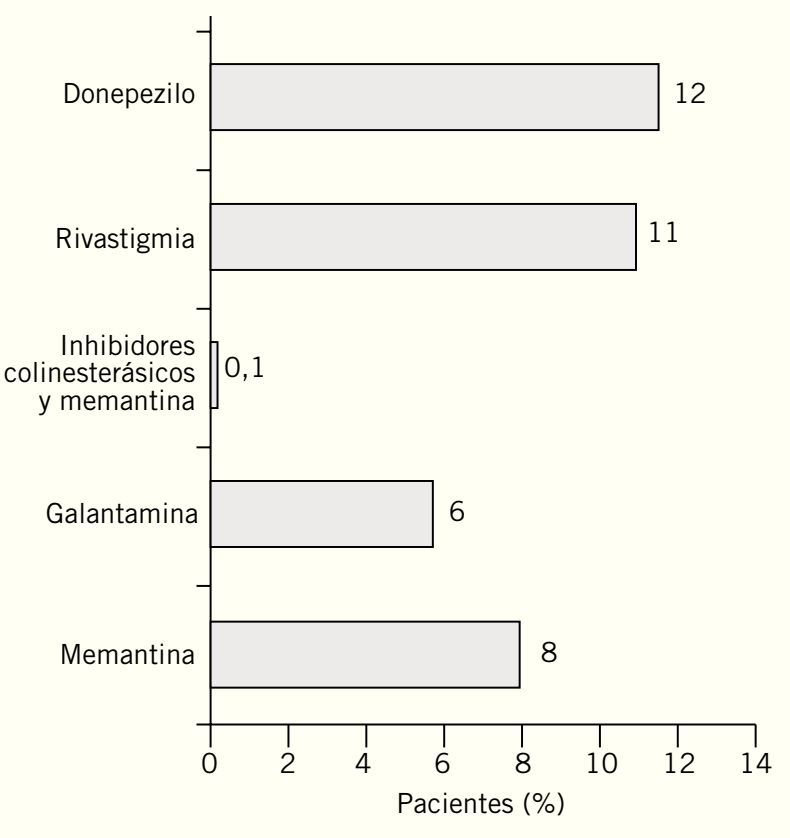

Concretamente el donepezilo es el principio activo que presenta una mejor tolerabilidad para los pacientes demenciados, ya que tienen más persistencia del tratamiento que otros principios activos como la rivastigmina o la galantamina ${ }^{15}$. Sin embargo, el escalado lento de rivastigmina mejora su tolerabilidad y la llegada a dosis mayores (más terapéuticas) ${ }^{16}$. Por otra parte, una elevada proporción de los pacientes son tratados con fármacos neurolépticos (antipsicóticos), entre los que se encuentran la olanzapina y la risperidona. El tratamiento con olanzapina ha demostrado dar lugar a un aumento de la mortalidad de los pacientes demenciados debido a un aumento del riesgo de accidente cerebrovascular. Se ha comunicado que este efecto podría producirse también en el tratamiento con risperidona ${ }^{17}$, aunque esta afirmación es en exceso controvertida por la forma en que se ha llegado a ella. Siempre será preferible, en caso de necesidad, utilizar los neurolépticos atípicos que los típicos, ya que el riesgo de enfermedad cerebrovascular es similar ${ }^{18,19}$ y los segundos causan un exceso de efectos secundarios. De hecho, la risperidona es el único fármaco que tiene aprobada esta indicación.

Aunque, en general, los pacientes son tratados con fármacos específicos para su proceso (inhibidores colinesterásicos), una gran proporción de los pacientes son tratados con neurolépticos, lo que da lugar a una falta de especificidad en el tratamiento que, en algunos casos, puede ser contraproducente por sus efectos secundarios. En este sentido está demostrada la utilidad de los IACE (en especial la rivastigmina) en la reducción de los trastornos de conducta y su utilidad en las demencias más frecuentes: enfermedad de Alzheimer, enfermedad vascular y de Lewy ${ }^{20,21}$. 
En general, no se puede decir que haya un tratamiento estándar para la demencia, sino que depende directamente del estado en que se encuentra el paciente y de los síntomas concretos que rodean la enfermedad 22 . Son múltiples los fármacos que se puede utilizar y deben ser prescritos de manera individualizada, lo que pone de manifiesto la necesidad de evaluación de los pacientes demenciados por un especialista que lleve a cabo un análisis general de la situación de cada paciente valorando los beneficios y riesgos que cada grupo farmacológico puede tener para el paciente y realizando un estrecho seguimiento.

Según los datos extraídos del estudio DECADA, en el conjunto del Estado español, el abordaje que se realiza en los centros es heterogéneo e independiente, lo que puede dar lugar a una diferente calidad en la atención de los pacientes en función del centro en el que sean atendidos. Se hace necesaria, pues, la elaboración y la implantación de guías de buena práctica clínica para el adecuado tratamiento de los pacientes demenciados.

Por otra parte, en opinión de los autores, el acceso de los pacientes con demencia a centros especializados atendidos por geriatras titulados es un requisito de calidad y de estructura que en muchas ocasiones no se cumple en la actualidad; sobre todo, si se considera a los pacientes con demencias avanzadas y, por tanto, con una gran comorbilidad que supera generalmente el campo de acción de otras especialidades concurrentes (neurólogos, psiquiatras, etc.) en el tratamiento de las demencias.

\section{Agradecimientos}

Este trabajo ha sido impulsado por el grupo de demencias de la Sociedad Española de Geriatría y Gerontología (SEGG) y por Novartis Farmacéutica, S.A. Los autores agradecen la participación de los vocales autonómicos de la SEGG, los investigadores y los centros del estudio. Este trabajo recibió el apoyo técnico de KM Health (GOC Networking).

\section{Bibliografía}

1. Mouronte Liz P, Sánchez Pascual P, Olazarán Rodríguez J, Buisán Pelay R. El apoyo a los cuidadores de pacientes con enfermedad de Alzheimer. Enfermería Científica. 2001;232 233:10-7.

2. Hipótesis sobre la evolución futura de la mortalidad [en línea]. Instituto Nacional de Estadística (INE), Agosto 2001 [citado 5 Feb 2007]. Disponible en: http://www.ine.es

3. American Psychiatric Association. Diagnostic and Statistical Manual of Mental Disorders. 4th ed. Text Revision. Washington: American Psychiatric Association; 2000.

4. Rayuela Rico A, Macías Fernández JA. Gerontopsiquiatría (área de conocimiento 11). En: Cervera Enguix S, Conde López V, Espino Granado A, Giner Ubago J, Leal Cercos C, Torres González $F$, editores. Manual del Residente de Psiquiatría [citado 29 Abr 2007]. Sociedad Española de psiquiatria. Disponible en: http://sepsiq.org/
5. Ruipérez I, Midón J, Gómez-Pavón J, Maturana N, Gil P, Sancho $\mathrm{M}$, et al. Nivel de adecuación de los recursos geriátricos en los hospitales generales españoles. Rev Esp Geriatr Gerontol. 2003;38:281-7.

6. Gil Gregorio P, Arriola Manchola E, Regidor García J, García Navarro JA, Ruipérez Cantera I. Presencia de especialista en geriatría en los centros que atienden a pacientes con demencia. Rev Esp Geriatr Gerontol. 2006;41:378-9.

7. Small GW, Rabins PV, Barry PP, Buckholtz NS, DeKosky ST, Ferris $\mathrm{SH}$, et al. Diagnosis and treatment of Alzheimer disease and related Disorders. Consensus statement of the American Association for Geriatric Psychiatry, the Alzheimer's Association, and the American Geriatrics Society. JAMA. 1997;278: 1363-71.

8. Snowdon DA, Greiner LH, Mortimer JA, Riley KP, Greiner PA, Markesbery WR. Brain infarction and the clinical expression of Alzheimer disease. The Nun Study. JAMA. 1997;227: 813-7.

9. Petrovitch H, White LR, Izmirilian G, Ross GW, Havlik RJ, Markesbery W. Midlife blood pressure and neuritic plaques, neurofibrillary tangles, and brain weight at death: the HAAS. Neurobiol Aging. 2000;21:57-62.

10. Boustani M, Peterson B, Harris R, Lux LJ, Krasnov C, Sutton FS. Screening for dementia. Systematic evidence review [en línea] Rockville (MD); Agency for Healthcare Research and Quality; 2003 (Systematic evidence review; n. ${ }^{\circ} 20$ ) [citado 5 Feb 2007]. Disponible en: http://www.ahrq.gov/downloads/pub/prevent/pdfser/dementser.pdf

11. Reisberg B, Ferris SH, De León MJ. The global deterioration scale for assessment of primary degenerative dementia. Am J Psychiatry. 1982;139:1136-9.

12. Boletín Oficial de las Cortes de Castilla y León. N.²6. Año XVII. 1 de diciembre de 1999.

13. Abordaje terapéutico de las demencias. ¿Qué pacientes podemos tratar? [en línea] El Médico Interactivo. Diario Electrónico de la Sanidad. Programa de Formación Continuada Acreditada para médicos de Atención Primaria, 2004 [citado 5 Feb 2007]. Disponible en: http://www.elmedicointeractivo.com/formacion_acre2004/tema4/demencias.htm

14. Segura T, Galindo MF, Rallo-Gutiérrez B, Ceña V, Jordán J. Dianas farmacológicas en las enfermedades neurodegenerativas. Rev Neurol. 2003;36:1047-57.

15. Sicras A, Rejas-Gutiérrez J. Patrón de persistencia de tratamientos anticolinesterásicos en la demencia de tipo Alzheimer: análisis comparativo retrospectivo del donepezilo, la rivastigmina y la galantamina. Rev Neurol. 2004;39:312-6.

16. Shue-Haim JR, Smith J, Amin S. Slow dose escalation of rivastigmine in the treatment of patients with Alzheimer's disease: an analysis of two prospective studies. Int Congress of AD. Poster P202. Stocholm; 2002.

17. Nota informativa de la Agencia Española de Medicamentos y Productos Sanitarios. Riesgos de olanzapina y risperidona en ancianos con demencia. Prescripción de fármacos. 2004;10: 30-1.

18. Gill SS, Rochon PA, Herrmann N, Lee PE, Sykora K, Gunraj N, et al. Atypical antipsychotic drugs and risk of ischaemic stroke: population based retrospective cohort study. BMJ. 2005;330: 445-51. 
Gil P et al. La demencia en España: evolución desigual entre las necesidades de los pacientes y la oferta asistencial desde la geriatría

19. Wang PS, Schneeweiss S, Avorn J, Fischer MA, Mogun H, Solomon $\mathrm{DH}$, et al. Risk of death in elderly users of conventional vs. atypical antipsychotic medications. N Engl J Med. 2005; 353:2335-41.

20. Farlow MR. Update on Rivastigmine. The neurologist. 2003; 9:230-4.
21. Fort I. Tratamiento farmacológico de la demencia. Rev Mult Gerontol. 2004;14:34-46.

22. Grossberg GT. Effect of rivastigmine in the treatment of behavioral disturbances associated with dementia: review of neuropsychiatric impairment in Alzheimer's disease. Curr Med Res Opin. 2005;21:1631-9. 